\title{
Surgical Facelift
}

\author{
Velupillai Ilankovan and Tian Ee Seah
}

\subsection{Introduction}

An ancient Chinese proverb “岁月不饶人” observed that "no one is spared from the ravages of ageing". A combination of age, gravity, physiologic contraction of facial muscles and solar (sun) and chemical (smoking) insults results in intrinsic and extrinsic changes to the skeleton, muscles and skin [1]. Preservation of one's youthful looks has been a goal of humans regardless of cultures and ethnicities. The search for this elixir of youth was found unexpectedly in the hands of cosmetic surgeons. Even with the recent advent of skin peels, botulinum toxin injections, lasers and injectable filler injections, the century-old facelift still possesses the most dramatic improvement to the moderate to severe ageing lower face when done well.

Facelift or rhytidectomy as its name suggests is a procedure to partially eliminate folds, creases and wrinkles (rhytids) caused by gravity and degeneration. In effect, the creation of two large cervicofacial flaps, which, after suspension and trimming, produces an overall tightening of the skin and the fascial envelope of the face and neck, results in restored anatomical structure. Facelift can help to negate some of these gravitational problems and produce some intrinsic improvement.

\subsection{Historical Perspective}

Historically, rhytidectomy was limited to skin elliptical excisions and tightening. The German surgeon, Eugen Höllander, first described being persuaded by his patient to remove excessive skin in the temporal, preauricular and postauricular regions in 1901. With cosmetic surgery frowned upon in

\section{Ilankovan $(\bowtie)$}

Oral and Maxillofacial Surgery, Poole Hospital NHS Foundation

Trust, Poole, UK

T. E. Seah

TES Clinic for Face and Jaw, Singapore, Singapore those times, he did not report his surgery immediately but only recounted retrospectively that the iatrogenic wounds when approximated resulted in some tightening in 1912 [2]. The initial reports of rhytidectomy were dominated by a slew of American surgeons such as Cantrell (1902), Miller (1907) and German surgeons, Lexer (1906) and Joseph (1912) [3]. The French surgeon Passot wrote an important paper "La chirugie esthétique des rides du visage" in 1919 where he illustrated strategically placed forehead, malar, cheek and submental elliptical incisions to tighten the face for aesthetic purposes, thus heralding the concept of short scar rhytidectomy (Fig. 37.1).

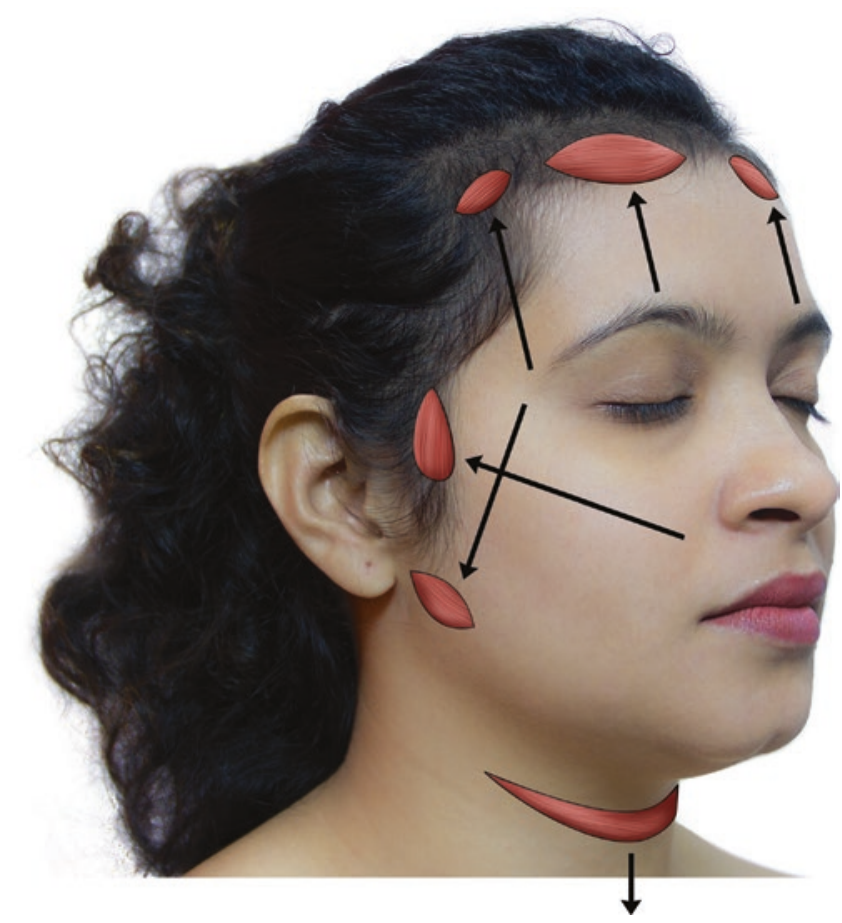

CAssociation of Oral and Maxillofacial Surgeons of India

Fig. 37.1 Illustration showing Passot's principles on facelift through elliptical excisions 
However, with limited undermining, wounds were closed under tension, resulting in unsightly scarring. To circumvent this problem, skin flap only procedures during which only the skin was raised, undermined for a short distance and tightened were introduced (Bames 1927) [4].

The results of skin flap only facelift were short term with limited correction of the ageing face, forcing surgeons to search for better alternatives. In 1974, Tord Skoog, a Swedish surgeon, revolutionized facelift concepts by describing the dissection, elevation and tightening of the superficial fascia of the face in addition to removing excess skin. He expounded the importance of retro-positioning the "buccal fascia" and the platysma for better results. The importance of this technique was later cemented when the superficial fascia of the face was clarified as the "superficial muscular aponeurotic system" (SMAS) by Mitz and Peyronie in a landmark paper in 1976 [5]. Manipulation of the SMAS layer in a superior or superolateral vector became an important part of modern facelift. Soon, a flurry of papers on the modifications to facelift procedures concentrated on the management of the SMAS.

Treatment of the SMAS layer could be broadly categorized into three methods. They were plication, elevation, imbrication and SMASectomy [6]. The most conservative of these methods was plication of the SMAS layer, as it did not actually involve dissecting the SMAS layer but instead used sutures to fold the SMAS and achieve a tightening effect [7]. More aggressive management of the SMAS included imbrication, which involved the elevation and repositioning of the SMAS in a superior or superolateral vector [8]. In standard SMAS elevation, the SMAS was dissected, lifted off the parotid fascia and secured to the underlying zygomatic soft tissues or the deep temporal fascia (Fig. 37.2).

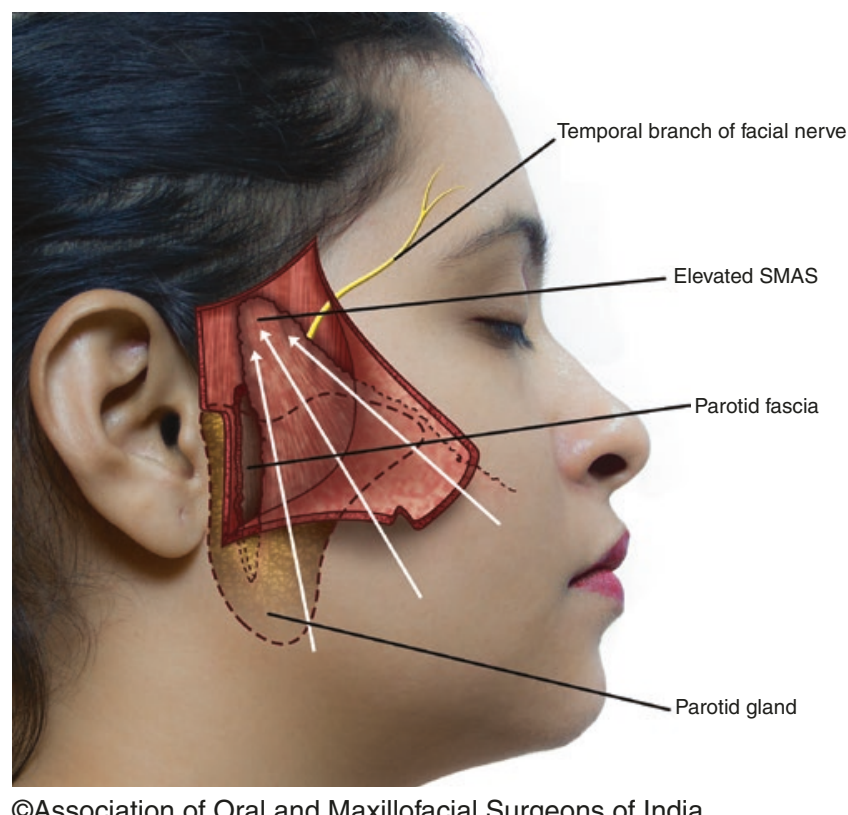

(CAssociation of Oral and Maxillofacial Surgeons of India

Fig. 37.2 SMAS imbrication

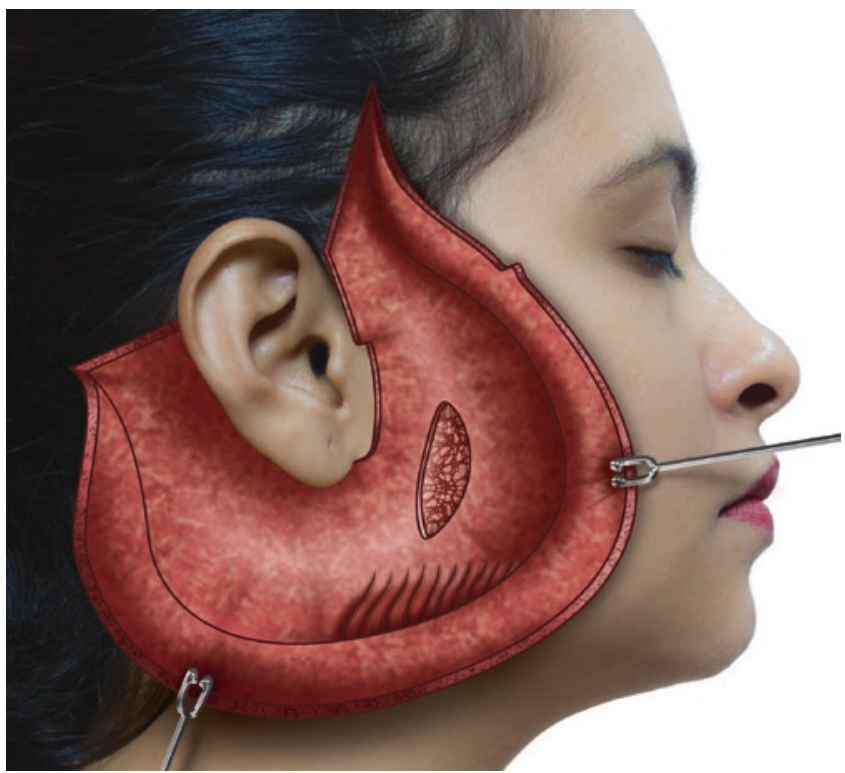

(C)Association of Oral and Maxillofacial Surgeons of India

Fig. 37.3 SMASectomy. 1-2 cm of the SMAS is excised superficial to the anterior border of the parotid

SMASectomy was later described [9]. This required excision of approximately $1-2 \mathrm{~cm}$ strip of the SMAS, superficial to the underlying parotid fascia, and then tightening were carried out by suturing the opposing two cut SMAS margins [6] (Fig. 37.3).

It soon became obvious to the surgeons that skin excision and SMAS manipulation were only effective in treating the lower face but not as effective in treating midface ageing. In particular, the nasolabial fold proved to be a stubborn adversary. The spotlight turned towards the high density, retaining ligaments such as the zygomatic retaining ligaments, which prevent effective rejuvenation of the midface using the aforementioned procedures.

The deep plane facelift was described by Hamra to include the malar fat pad [10]. In this, the dissection and elevation of the SMAS layer were carried out further to release the zygomatic retaining ligaments so that more traction could be obtained from the midface. The orbicularis oculi and later septal reset were included to improve midface aesthetics, and the term composite facelift was coined by Hamra [11].

As the facelift landscape progressed, efforts turned almost full circle towards smaller incisions. Comparisons of different published facelifts were unable to reveal an optimal technique [12] although complications were significant higher in extensive SMAS procedures.

Attention turned towards having minimal excision, limited dissection and SMASectomy with imbrication, and in 2001, Saylon and later Fulton et al. described the S-lift [13]. In this technique, the preauricular skin was excised in a predetermined S-shape followed by purse-string plication of the 
mobile SMAS using a U-shaped purse string suture and an $\mathrm{O}$-shaped purse string suture. Both sutures were anchored into the periosteum of the zygoma.

Tonard et al. later introduced the minimal access cranial suspension (MACS) as a modification to the S-lift [14] (Fig. 37.4).

In this procedure, he described suspending the sagging SMAS tissues with cranially directed purse string sutures via limited incisions that extend from the sideburn to the inferior helical attachment. Four main differences lie between the "S" lift and the 'MACS' lift. First, the S-Lift pre-empted the amount of excess skin and excised it via an S-shaped incision. The MAC lift redraped the excessive skin and removed it according to the margin. The second difference lies between point of anchorage as the 'S-lift' anchored to the periosteum of the zygoma, while the 'MACS lift' anchors to the superficial layer of the deep temporal fascia. Third, during the S-lift, SMASectomy and imbrication were carried out, while during the MACS lift, only plication was performed. Fourth, besides the two $\mathrm{O}$ and U-shaped sutures, the MACS lift utilizes a third vertical suspension suture that suspended the malar fat pad, thus reducing the depth of the nasolabial fold. This area is accessed by extending the temporal hairline incision, and Tonard and Verpaele called this the extended MACS lift.

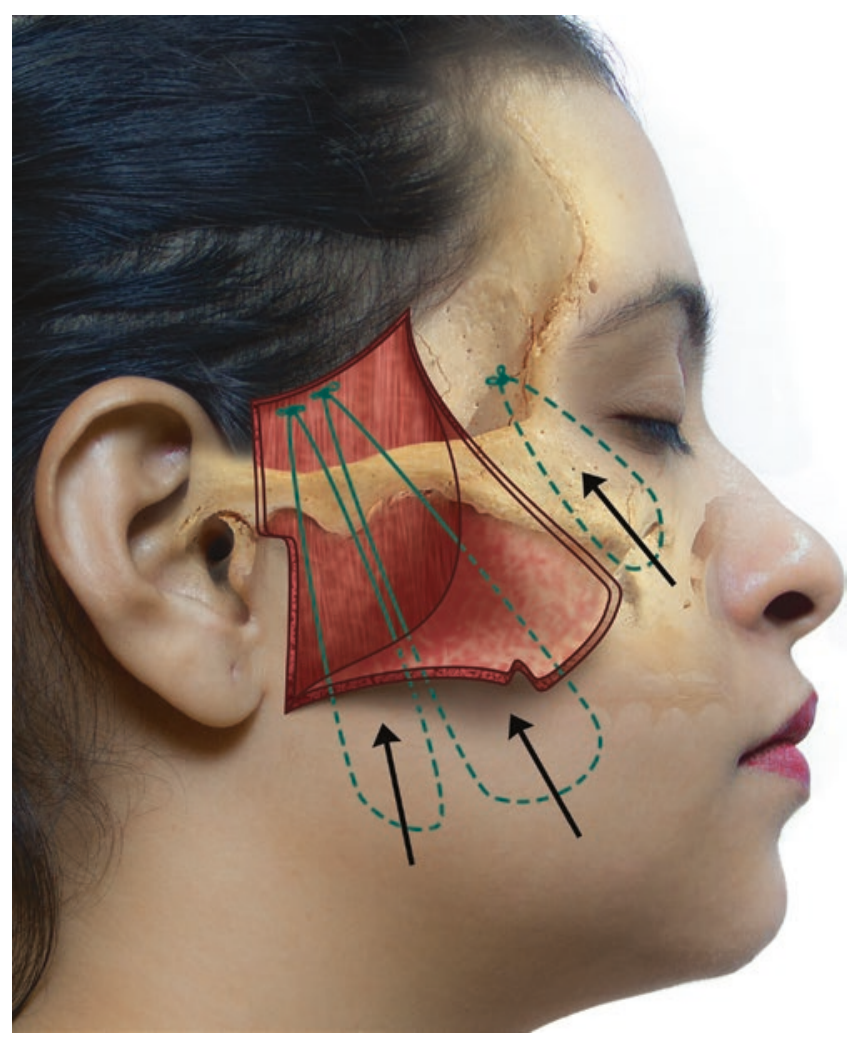

(CAssociation of Oral and Maxillofacial Surgeons of India

Fig. 37.4 MACS Lift

\subsection{Anatomy of Ageing Face}

Some of the gravitational changes include lateral brow ptosis, tear trough, malar palpebral groove, SOOF descent, malar fat descent, deepening of the nasolabial groove, buccal fat pad herniation, marionette lines, jowls, loss of neck definition, submental fat and excess skin [1].

With ageing, a combination of gravitational forces, laxity of the skin due to loss of elasticity, decreased dermal thickness and loss of dermal appendages results in ptotic, hanging skin.

The mid cheek's fat is divided into three compartments [15] (Fig. 37.5). It is thinnest at the lid-cheek segment and is thickest in the nasolabial region. The malar fat is also moderately thick although not as thick as the nasolabial region.

Accumulation of fat in the cervical region results in loss of neck definition and submental fat.

Superficial fat and deep fat are separated by SMAS layer. The superficial fat is separated into five compartments, namely, the nasolabial, medial cheek, middle cheek,

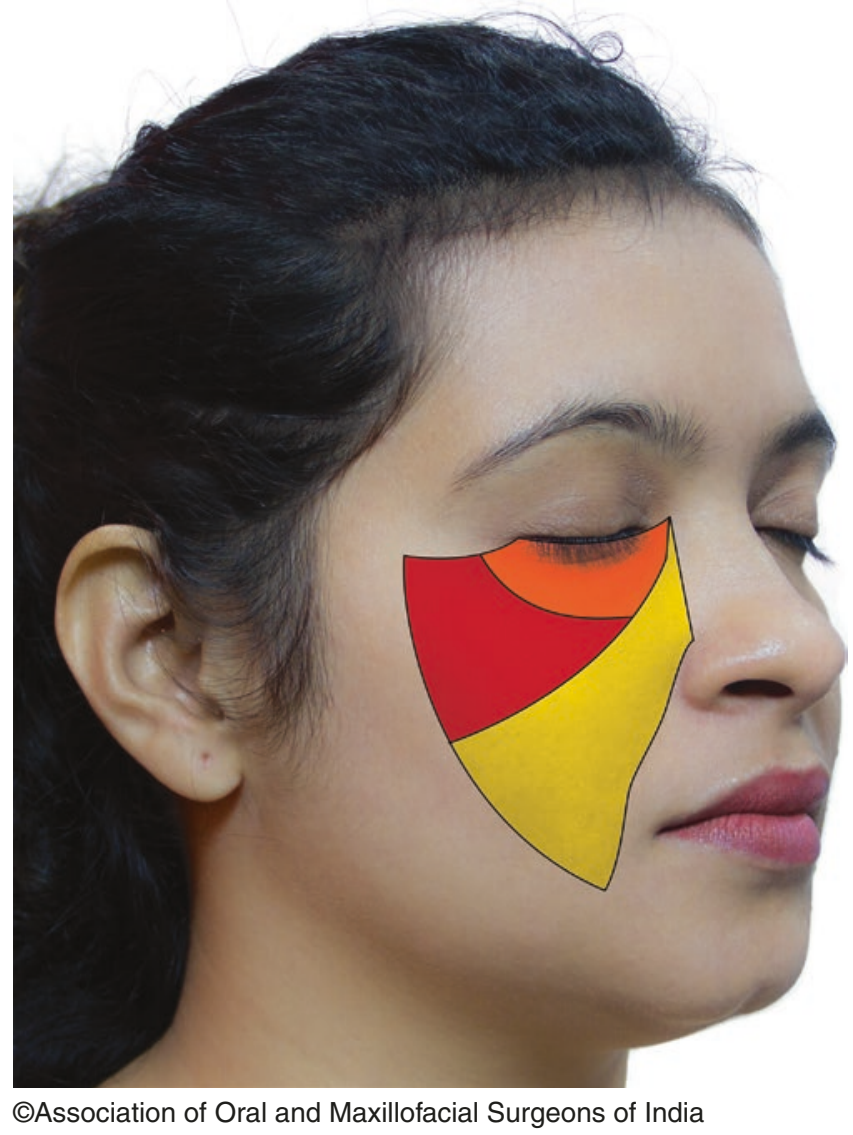

Fig. 37.5 Cheek fat is divided into three compartments. They are lidcheek segment (Orange), Malar segment (red) and nasolabial region (yellow). (Figure adapted and redrawn from Aesthetic Plastic Surgery 2009, Chapter 6 by Mendelson BC) 
lateral temporoparietal and inferior orbital fat. Deep fat is divided into the deep medial fat and suborbicularis oculi fat (SOOF) [16].

The superficial musculoaponeurotic system (SMAS) is a fibrous sheath, which was described in a classical paper by Mitz and Peyronie [5] in 1976, and starts from the galea superiorly. Superolaterally, it is continuous with the superficial temporal fascia also known as the temporoparietal fascia. In the central upper face, deep into the superficial fat, the SMAS layer is invested by the muscles of facial expression, derived from the embryonic second branchial arch: frontalis, orbicularis oculi, corrugator supercilii, depressor supercilii and the procerus. In the middle third, the SMAS is invested by the nasalis muscles, zygomaticus major, minor, levator labii superioris alaeque nasi and levator labii superioris. Laterally, the SMAS is superficial to the parotid fascia and extends inferiorly to include the platysma muscles. Inferomedial, the SMAS is invested by the orbicularis oris, risorius, depressor anguli oris, depressor labii inferioris and mentalis and is continuous with the platysma.

With the exception of the levator anguli oris and mentalis, the facial nerve travels deep into the SMAS layer and innervates the muscles of facial expression, making dissection above the SMAS during facelift safe [16].

An analogy of the retaining ligaments is that they are rooted in a tree into the periosteum and deep fascial thickening, and as it approaches the SMAS, it divides into numerous branches and inserts into the dermis called retinacular cutis [15] (Fig. 37.6).

Stuzin [17] and Furnas et al. [18] described the retaining ligaments [17]. The partitioning of separate fascial spaces and compartments is caused by these retaining ligaments, which are classified into osteocutaneous and fasciocutaneous retaining ligaments.

Osteocutaneous retaining ligaments originate from the periosteum and include the zygomatic and mandibular cutaneous ligaments. Stout zygomatic retaining ligaments originate from the inferior border of the zygomatic arch towards the junction between the arch and the body and insert into the dermis of the skin as fibrous septa. It is posterior to the zygomaticus major muscle, approximately $3 \mathrm{~mm}$ in width, $0.5 \mathrm{~mm}$ in thickness and $4.5 \mathrm{~cm}$ anterior to the tragus [18]. Loss of support results in malar fat descent.

The mandibular ligament originates from the periosteum $10 \mathrm{~mm}$ above the mandibular border, extends along the anterior one third of the mandibular body and inserts into the dermis [19] (Fig. 37.7). It coincides with the anterior margin of the jowl.

Fasciocutaneous retaining ligaments include the masseteric and parotid cutaneous ligaments also known as the platysma auricular ligament and coalesce between the superficial and deep fascia of the face [1].

Masseteric ligaments originate from the parotidomasseteric cutaneous ligaments, and- loss of support results in

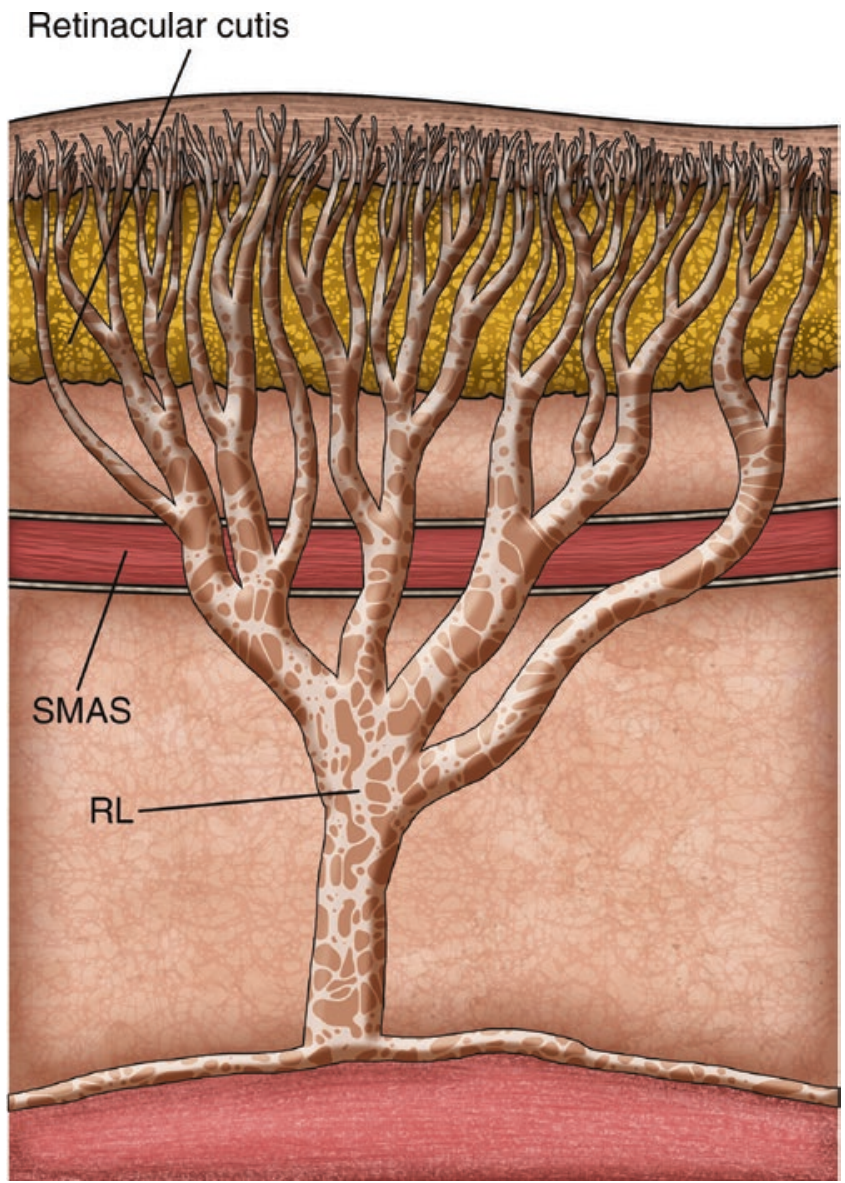

Fig. 37.6 Retaining ligaments (RLs) insert into the subcutaneous layer as retinacular cutis. It is likened to a tree by Mendelson BC [15]. (Figure adapted and redrawn from Aesthetic Plastic Surgery 2009, Chapter 6 by Mendelson BC)

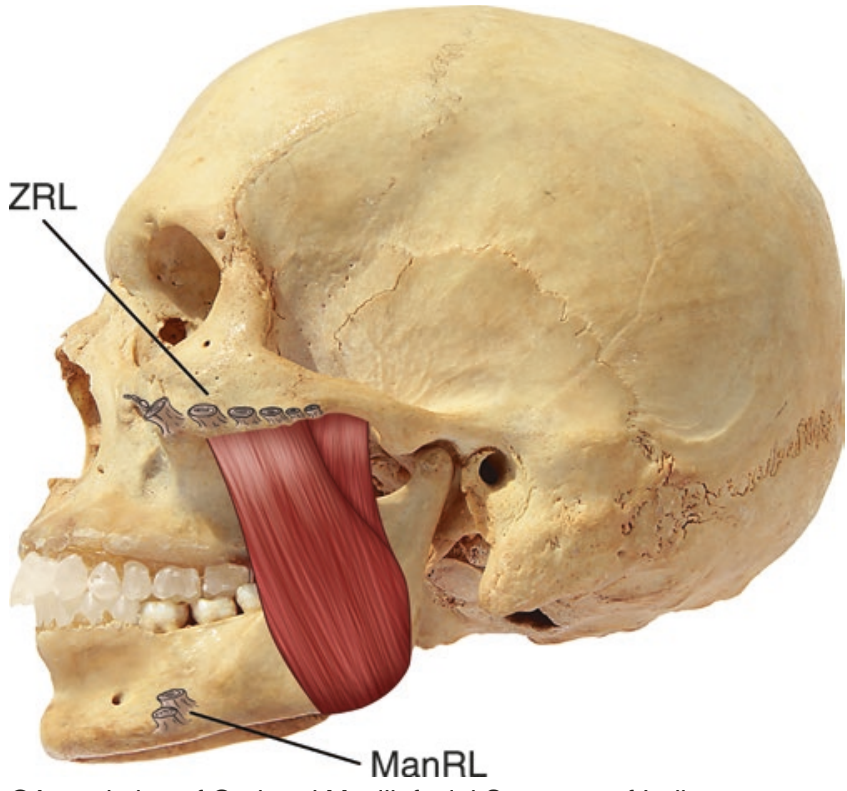

CAssociation of Oral and Maxillofacial Surgeons of India

Fig. 37.7 Osteocutaneous ligaments comprising the zygomatic retaining ligaments (ZRLs) and the mandibular retaining ligaments (MRLs) 


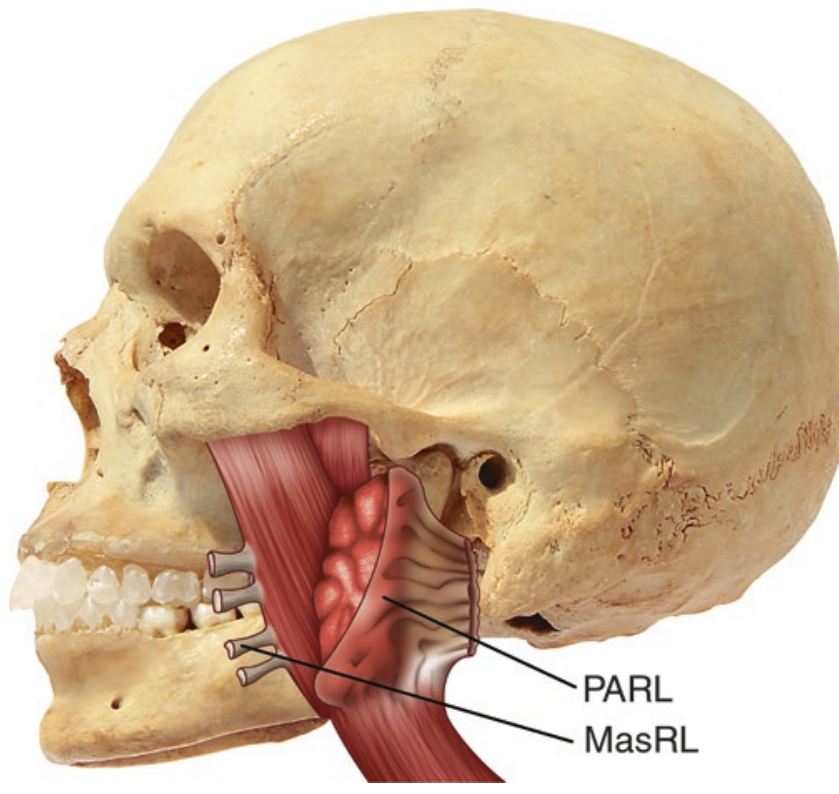

CAssociation of Oral and Maxillofacial Surgeons of India

Fig. 37.8 Fasciocutaneous retaining ligaments comprising the masseteric retaining ligaments (MasRLs) and the parotid cutaneous ligaments also known as platysma auricular retaining ligaments (PARLs)

facial jowling. Parotid cutaneous ligaments are formed by the posterior border of the platysma, which had receded into fascial condensation. It is fused with the parotid fascia, is attached to the overlying skin and provides firm anchorage between the platysma and the dermis in the inferior auricular region (Fig. 37.8).

\subsection{Standard Facelift}

Surgery can be carried out either under general anaesthesia or under local anaesthetic with or without sedation.

Local anaesthesia comprising Lidocaine $2 \%$ with adrenaline $1: 80,000$ is infiltrated in the zygomaticofacial region, infraorbital, mental, greater auricular (Fig. 37.9) and the lesser occipital nerves.

Tumescent solution reconstituted with $1 \mathrm{~L}$ of saline, $50 \mathrm{~mL}$ of $1 \%$ lidocaine, $1 \mathrm{~mL}$ of adrenaline $1: 1000$ and $6 \mathrm{~mL}$ of $8.4 \%$ Sodium Bicarbonate is then injected subcutaneously after stab incisions are placed in the temporal, preauricular, postauricular and submental regions. Approximately $300 \mathrm{~mL}$ of tumescent solution in all is injected.

In both approaches, infiltration of Tumescent solution in a subcutaneous plane is essential to carry out atraumatic dissection. In our practice, $95 \%$ of standard facelift are carried out with simultaneous neck lift, as we feel it would be impossible to mobilize the attenuated soft tissue to achieve symmetry and an unoperated look of the midface without bringing attention to the neck aesthetics.

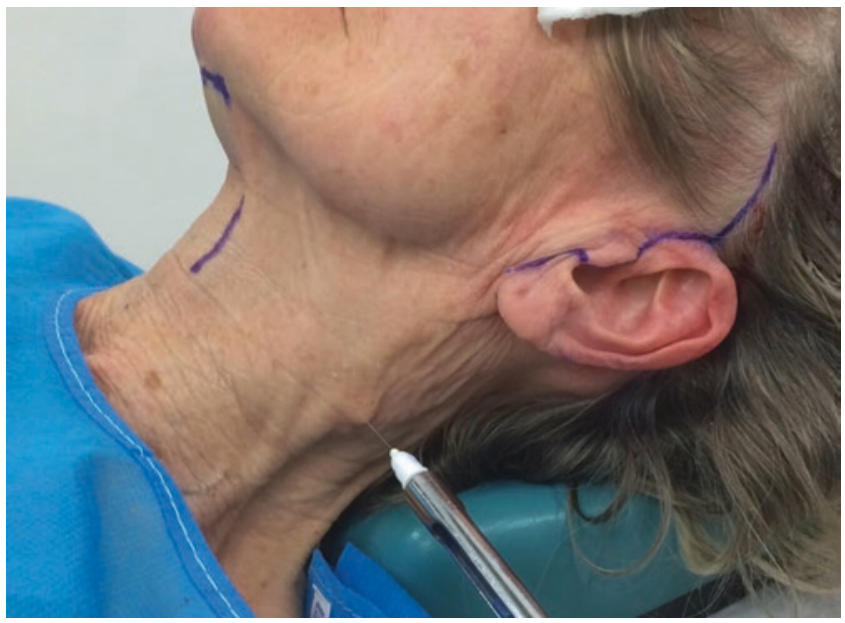

CAssociation of Oral and Maxillofacial Surgeons of India

Fig. 37.9 Greater auricular nerve block

\subsubsection{Incision}

The incision lines are divided into four sections: temporal, preauricular, postauricular and scalp extension. The temporal component is either into the hairline or just at the hairline/ sideburn border. The latter has the advantage of not moving the sideburn position; however, it would give a telltale sign of a visible scar. In the former approach, the sideburn area may get lifted into the temporal area and the scar will be hidden. This position should be made in conjunction with discussion with the patient, and suitability of the incision should be selected.

The preauricular marking could be a pre-tragal, intratragal or post-tragal. The latter can distort the tragal projection, and the pre-tragal will give a visible scar. We prefer an intra-tragal approach and obtain the tragal projection by placing a subcutaneous anchor suture before the final closure in order to maintain the natural concavity and simultaneously achieve an invisible scar (Figs. 37.10 and 37.11).

The postauricular incision should be a few millimetres into the postauricular area from the sulcus so that postoperatively the scar will settle down comfortably into the sulcus. The scalp extension is carried out by multiple $\mathrm{W}$ or $\mathrm{Z}$ plasty type incisions in a trichophytic fashion to minimize hair loss and to have an invisible scar (Fig. 37.12).

A $2.5 \mathrm{~cm}$ curvilinear incision parallel to the mandibular contour is the submental incision to the neck lift section in order to expose the anterior border of the platysma and the platysmal dissection.

\subsubsection{Dissection}

The submental flap dissection is carried out at first to expose the platysma muscle as much as possible (Fig. 37.13). 


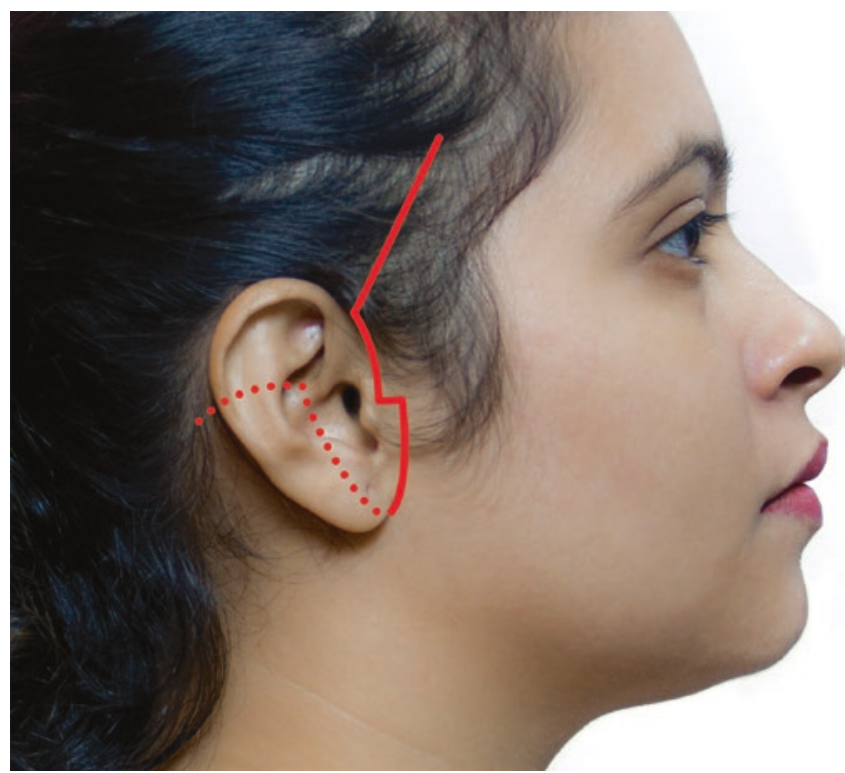

CAssociation of Oral and Maxillofacial Surgeons of India

Fig. 37.10 Temporal to preauricular incision (red). Occipital or scalp extension (broken line)

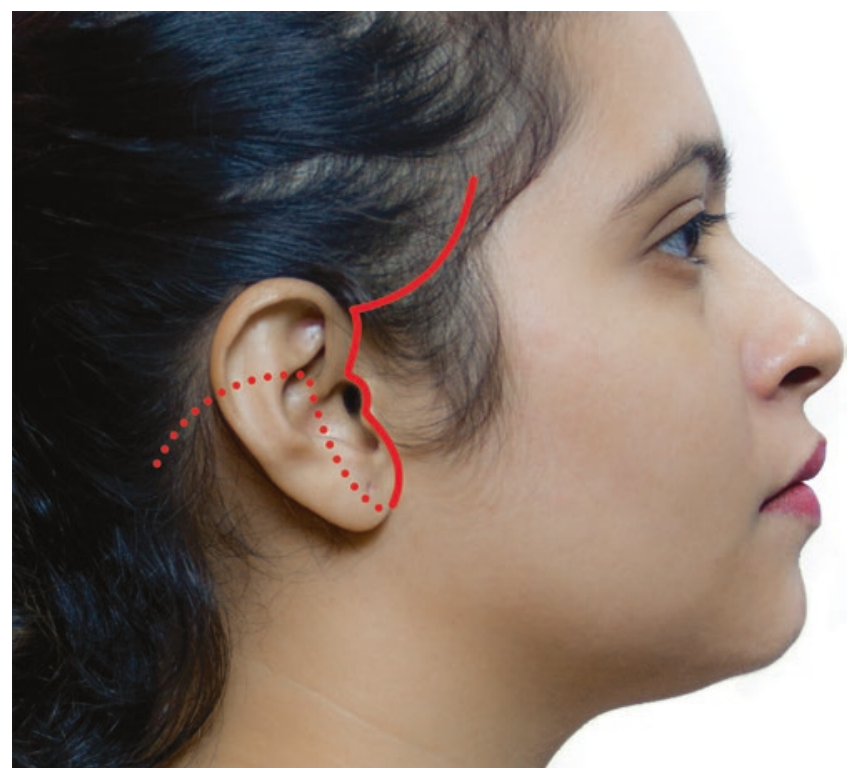

CAssociation of Oral and Maxillofacial Surgeons of India

Fig. 37.11 Hairline to tragal incision (red). Occipital or scalp extension (broken line)

Next, the scalp and postauricular dissection is carried out. The anterior border of the sternocleidomastoid muscle and posterior border of the platysma are exposed, and a cavity of the neck is connected anteriorly and posteriorly (Fig. 37.14). The next step is to focus on the temporal and preauricular dissection. In the temporal, the deep part of deep temporal

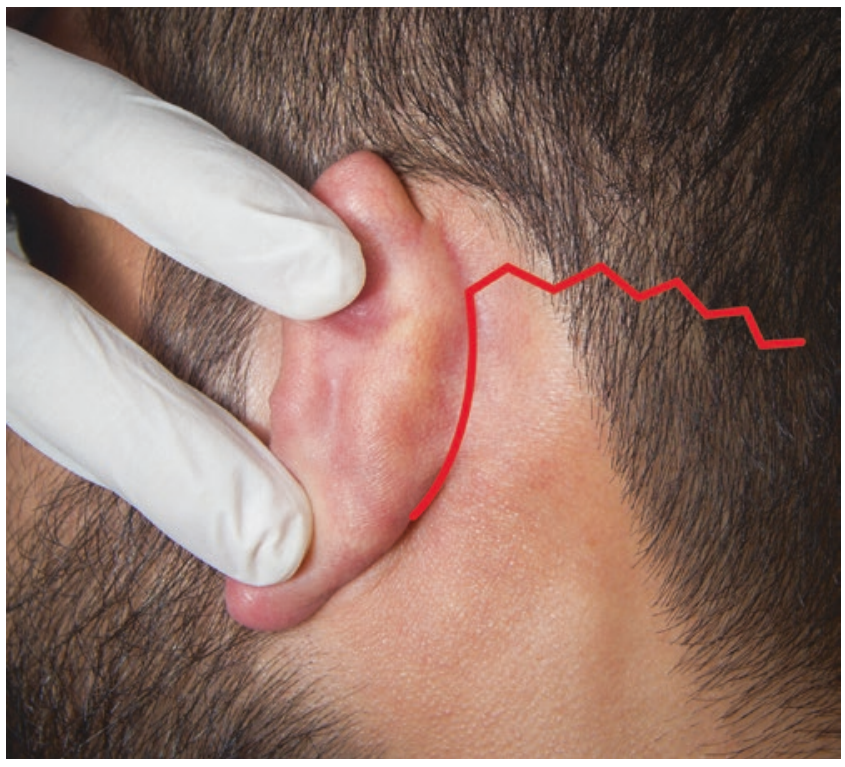

CAssociation of Oral and Maxillofacial Surgeons of India

Fig. 37.12 Illustration showing the postauricular and occipital or scalp extension

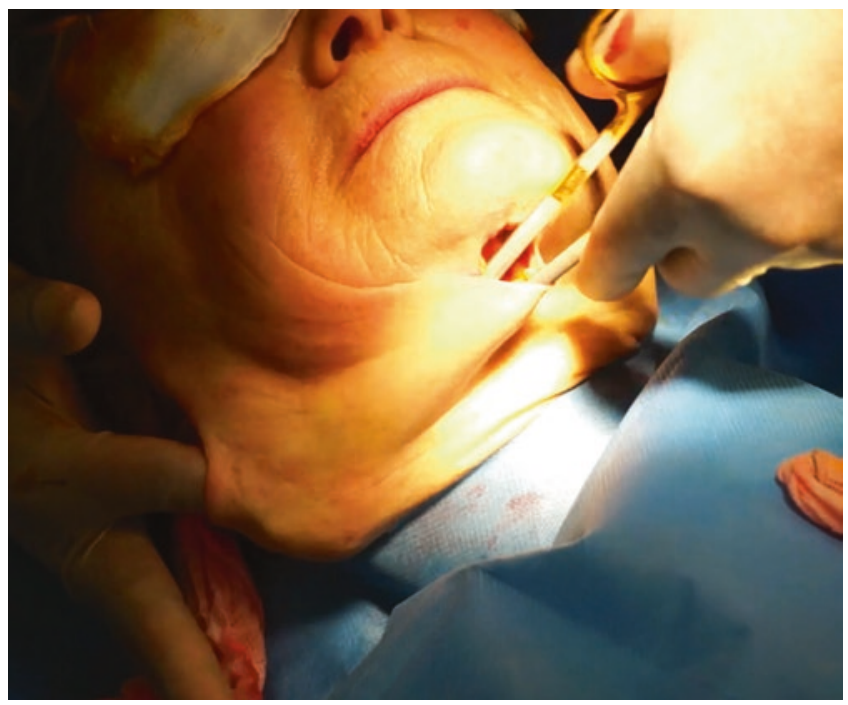

CAssociation of Oral and Maxillofacial Surgeons of India

Fig. 37.13 Dissection is in the supraplatysma plane

fascia is exposed, which we use as an anchor point to the SMAS suturing (Fig. 37.15). The preauricular dissection will expose the zygomatic ligaments, lateral end of the orbicularis oculi and the anterior border of the parotid-masseteric fascia with careful attention towards the buccal branch of the facial nerve (Fig. 37.16).

The preauricular, neck and postauricular dissection should now be in one cavity. 


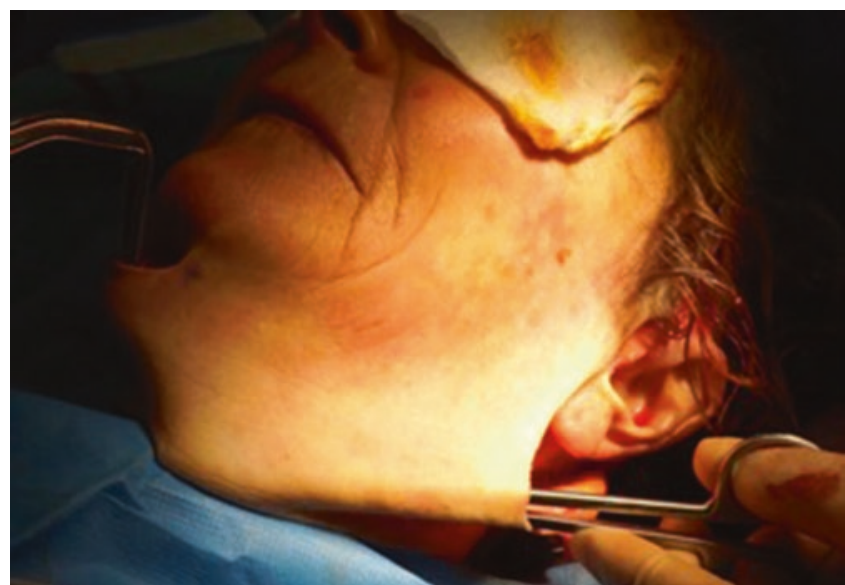

CAssociation of Oral and Maxillofacial Surgeons of India

Fig. 37.14 The postauricular flap is then connected to the cervical flap and the submental region made previously

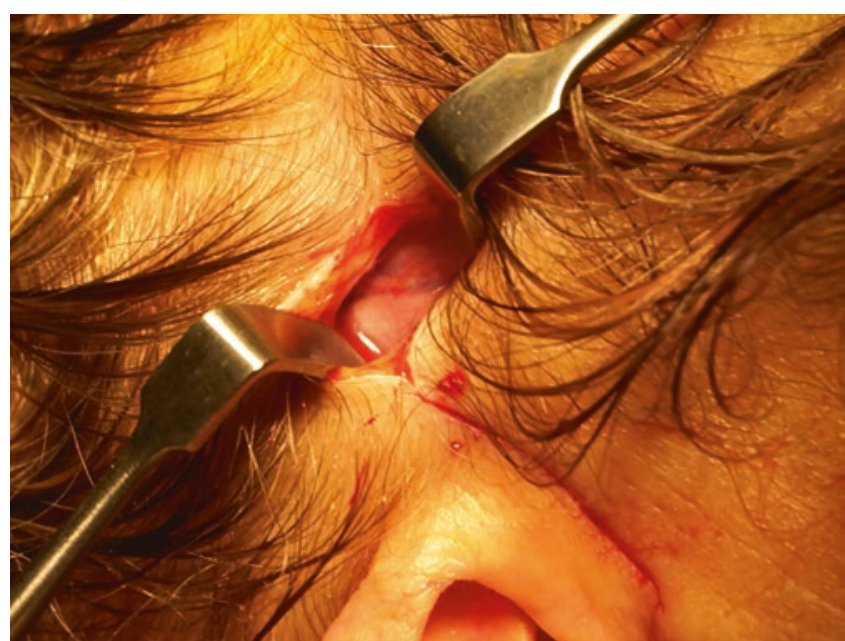

CAssociation of Oral and Maxillofacial Surgeons of India

Fig. 37.15 Deep layer of the deep temporal fascia exposed

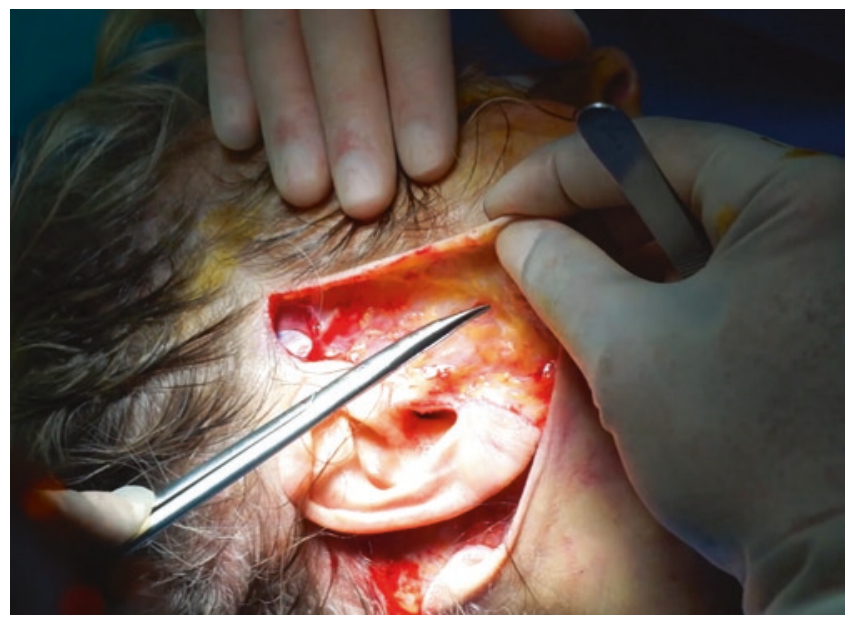

CAssociation of Oral and Maxillofacial Surgeons of India

Fig. 37.16 After the deep temporal fascia is exposed, dissection is carried out in a different plane subcutaneously to raise a skin flap

\subsubsection{SMAS and Platysmal Elevation and Plication}

SMAS elevation and management are carried out in various forms. We elevate the SMAS in an inverted $\mathrm{L}$ fashion (Fig. 37.17) starting at the preauricular plane, and the superior limit is at the lateral end of the zygomatic arch. The anterior dissection passes to the parotid-masseteric fascia (Fig. 37.18). The inferior dissection is to join the subplatys-

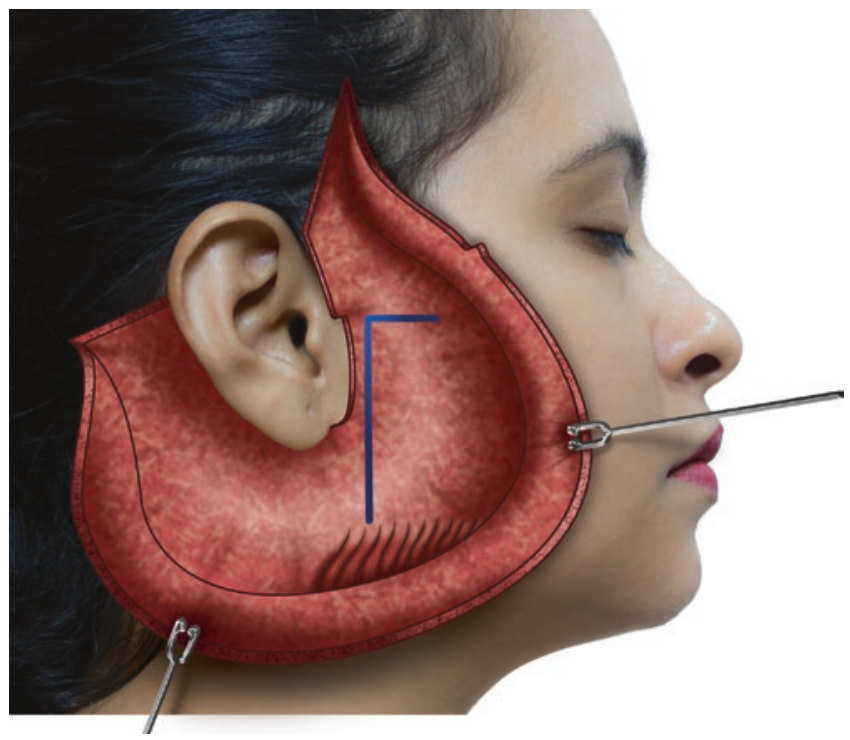

(C)Association of Oral and Maxillofacial Surgeons of India

Fig. 37.17 An inverted L shape incision (blue lines) is made on the SMAS stopping short of the parotid fascia. The shorter limb is just below the zygomatic arch, while the longer limb is in the preauricular region

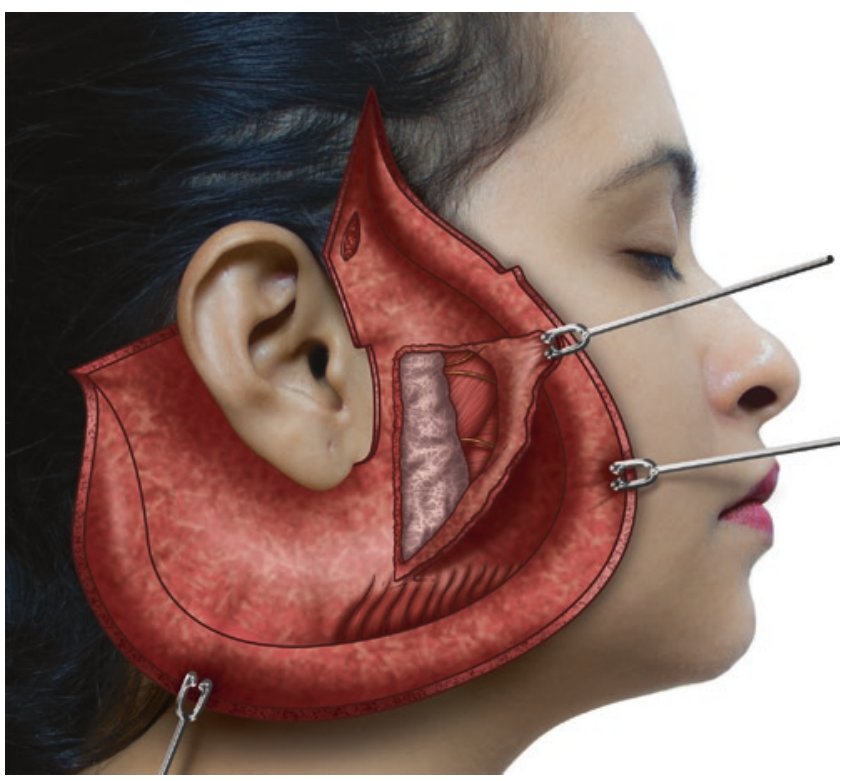

CAssociation of Oral and Maxillofacial Surgeons of India

Fig. 37.18 Cut SMAS peeled anteriorly to show underlying parotid. Facial nerve exits the parotid deep into the SMAS. Deep into these are the masseter muscles followed by the buccinator muscles with horizontal muscle striations 
mal plane at the angle of the jaw with consideration to avoid damage to the two or three cervical branches. In our practice, the elevated SMAS is anchored in a superior direction to the deep part of the deep temporal fascia (Fig. 37.19).

Our platysma dissection and plication are in four steps. The anterior border of the platysma is sutured in the midline [20] (Fig. 37.20). In some patients with short neck, the platysmal suturing needs to be hitched to the body of the hyloid bone. In some patients, a release of the inferior border of the platysma muscle at this area could also be considered. Then,

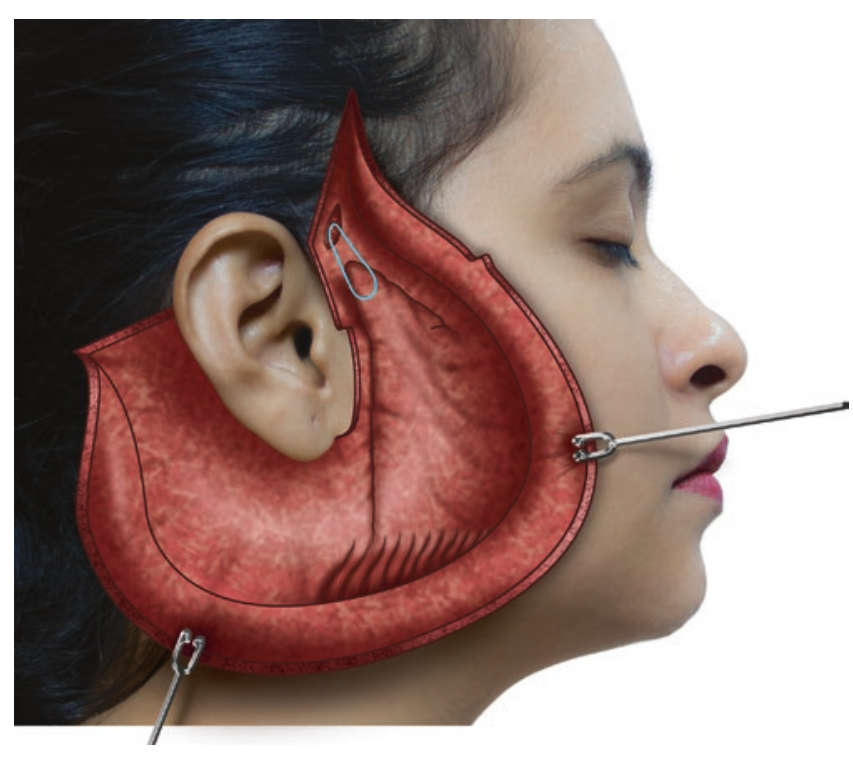

(C)Association of Oral and Maxillofacial Surgeons of India

Fig. 37.19 The cut SMAS is then advanced superiorly and sutured to the deep temporal fascia with 3/0 Polygluconate (Maxon, Tyco Health UK Ltd., Gosport PO130AS, UK) (blue line)

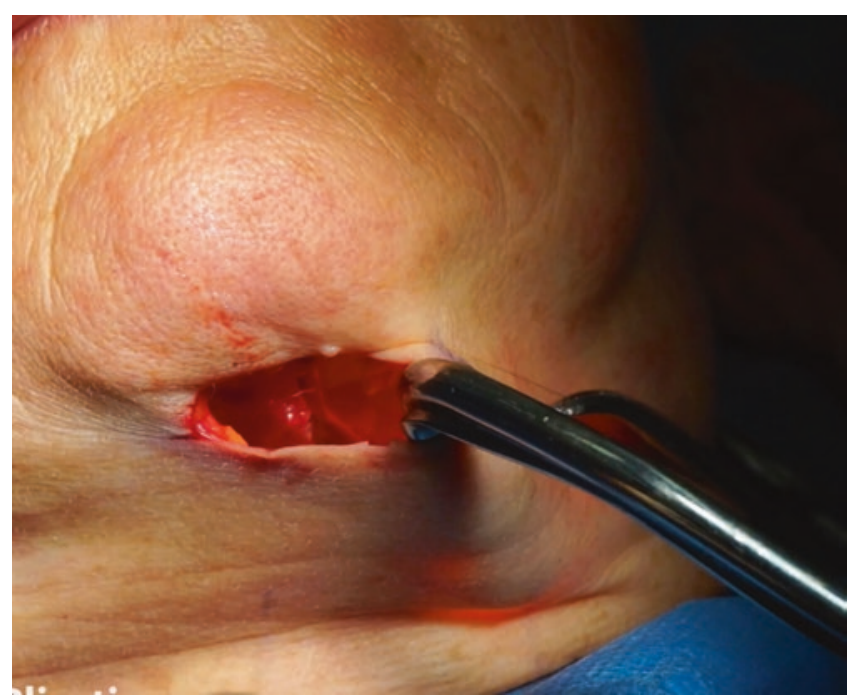

(C)Association of Oral and Maxillofacial Surgeons of India

Fig. 37.20 The medial edges of the platysma are sutured to each other at the midline in a corset manner

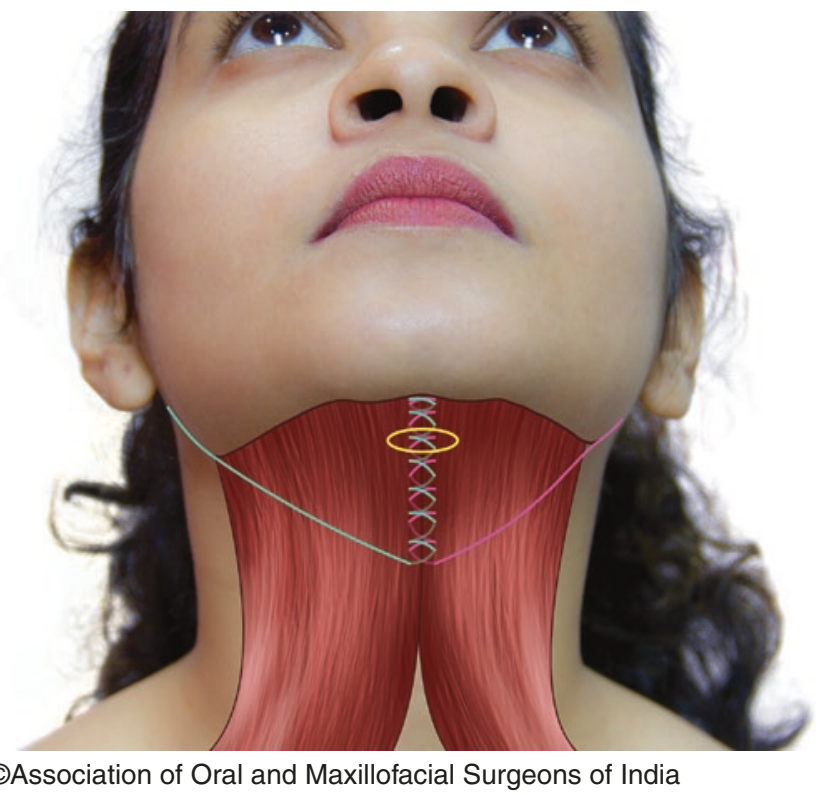

Fig. 37.21 A submental incision is made (white outline). Corset sutures are done twice, and the suture is plicated to the mastoid fascia on both sides

the lateral border of the platysma muscle is sutured to the anterior border of the sternocleidomastoid muscle. The third step is in platysmal plication is placing a cinch suture, which is placed joining the two anterior borders of the muscles in the midline and, after catching the platysma muscle in two or three areas, hitched the sternocleidomastoid muscle and the final anchor into the mastoid periosteum (Fig. 37.21). Depending on the neck anatomy, we sometimes place a second cinch suture about $5-6 \mathrm{~mm}$ below the first to avoid bulging of the submandibular gland.

\subsubsection{Closure}

Meticulous haemostasis is done with bipolar electrocautery throughout the procedure. The cervicofacial flaps are draped over the margins in a superolateral vector with much emphasis on the superior than the lateral direction (Fig. 37.22). Final anchoring is done with two key sutures that are placed along the helical attachment and the superior aspect of the postauricular incision. Cuts are made on the excess skin, perpendicular to the flap margin, stopping just short of it (Fig. 37.23). This allows the margins to be visualized when trimming the excess skin. If a tragal incision was made, the skin can be thinned and trimmed to mimic tragal shape and skin during closure at the tragus. Dog ears are preferably removed at the apex rather than in the ear lobe region. The ear lobe is then replaced $15^{\circ}$ posterior to the vertical position to reduce the telltale signs of facelift procedures. Layered repaired is then carried out in the final closure. 


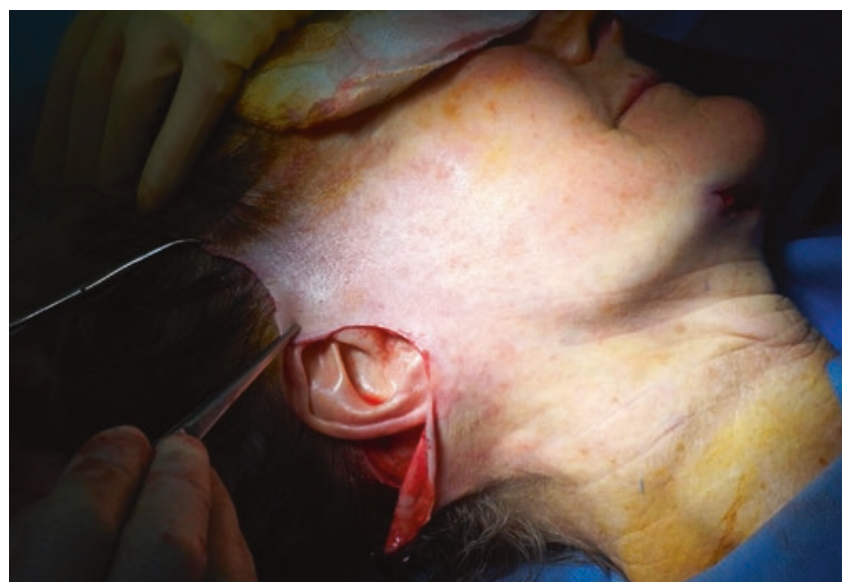

CAssociation of Oral and Maxillofacial Surgeons of India

Fig. 37.22 Excess skin is draped in a superolateral vector over the margins and excised

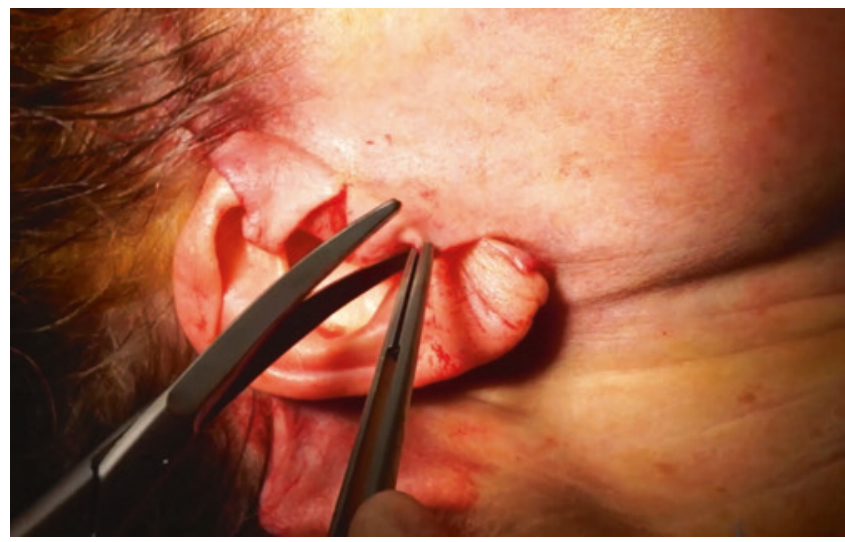

CAssociation of Oral and Maxillofacial Surgeons of India

Fig. 37.23 Cuts are made perpendicular to the flap margin stopping short of the margins and then excised accordingly

\subsubsection{Support Dressings}

There are different ways of providing this final exercise. We apply antibiotic topical ointment followed by non-sticky dressing to the pre- and postauricular area and an adjustable Velcro band. In the literature, however, various pressure dressings are applied.

\subsection{Post-operative Management}

Velcro bands are removed on a daily fashion. Wounds are cleaned followed by topical ointment and reapplication of the Velcro band.
The medications are taken on a prescribed fashion.

The patient is placed on antibiotics and painkillers for a week. Sleeping is done with the head elevated by $2-3$ pillows. The patient is seen on the day after the surgery to ensure that there is no haematoma. If drains are placed, they are removed during this time.

Facial bandages are placed between 2 and 5 days. Sutures are removed 7-10 days later. The wounds are kept moist with antibiotic ointment such as Tetracycline $3 \%$ or Mupirocin ointment 2\% (Figs. 37.24a, b and 37.25a, b).

\subsection{Complications and Management}

Post-operative complications of facelifts include postoperative haematoma, skin necrosis, sensory and motor disturbances, obvious scarring, seroma, alopecia, auricular deformity and dyspigmentation [21]. Detailed informed consent should be obtained from patients prior to the surgery.

\subsubsection{Haematoma}

A thorough medical history is needed prior to surgery. Antiplatelet medications such as Aspirin, Clopidogrel, Dipyridamole and powerful anticoagulation drugs such as warfarin will have to be stopped after consultation with the patient's prescribing physicians. Patients on herbal or traditional Chinese medications such as ginkgo biloba and Cordyceps will also need to be stopped before surgery.

Haematoma formation is by far the most common complication with some papers reporting up to $15 \%$ [21]. Expanding haematoma usually happens in the first 24 hours and must be evacuated. Smaller haematoma can be aspirated, and pressure bandage is placed for pressure haemostasis. Meticulous haemostasis with bipolar diathermy is mandatory for facelift procedures. The flap and the underlying bed should be scrutinized for bleeding spots with a good head light or fibre optic light attached to a retractor. Brisk bleeding from larger vessels should be ligated. Adjunctive measures including fibrin glue [22, 23] and platelet gel and facelift bandages have been explored.

Large haematoma are a cause for concern and need to be evacuated. They can cause problems in healing including skin ischaemia. 


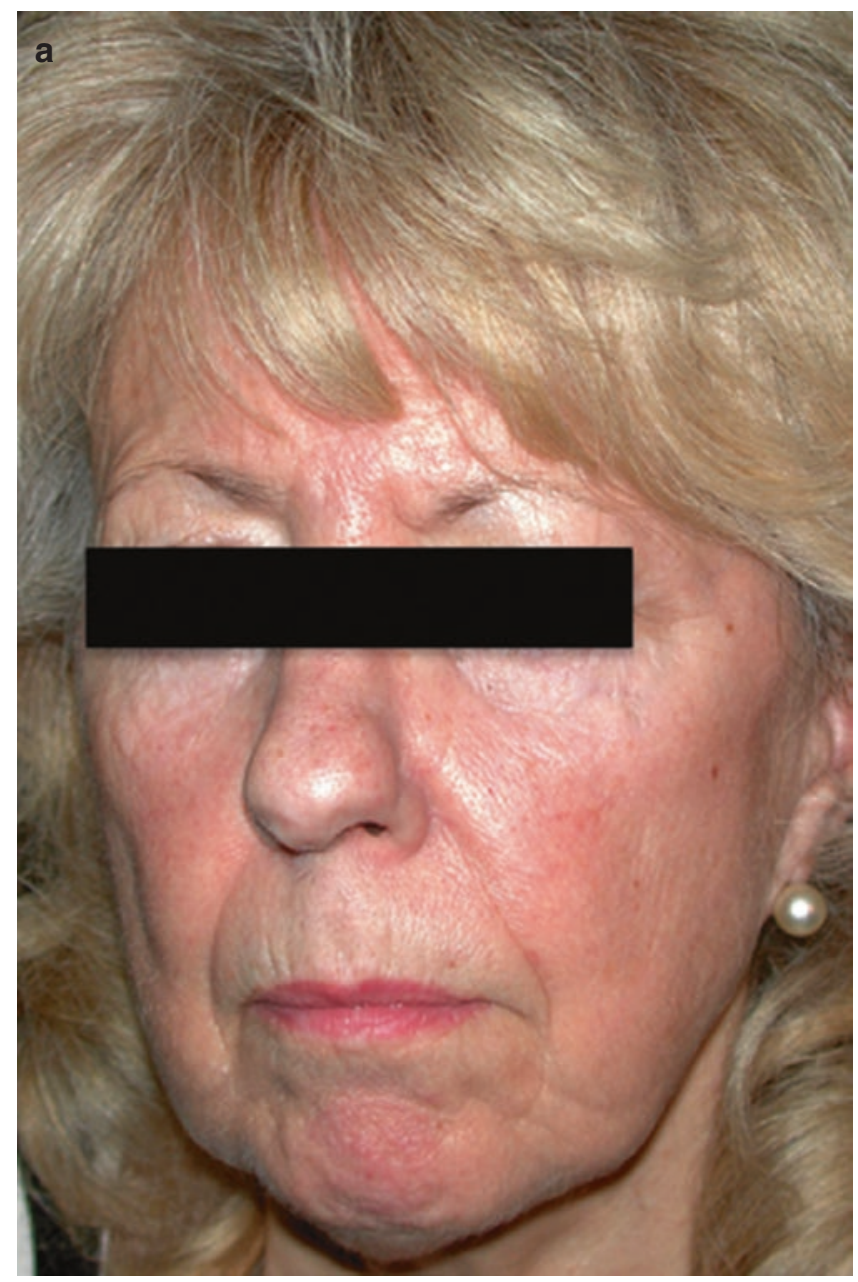

CAssociation of Oral and Maxillofacial Surgeons of India

Fig. 37.24 (a) Pre-operative profile view showing excess loose skin, jowling, submental fat and deep nasolabial fold. (b) Post-operative profile view after facelift and platysma plication. Excessive skin had been

\subsubsection{Skin Ischaemia and Necrosis}

Ischaemic changes and flap necrosis can occur due to circulatory disturbances. This can happen when the flap is too thin or had underwent extensive undermining and exuberant cautery, closed under excessive tension or compromised by excessively tight bandage compression. The incidence ranges between 1.1 and 3\% [24]. Venous congestion or arterial obstruction can result in skin ischaemia and necrosis. Large unevacuated haematoma separates the flap off the underlying

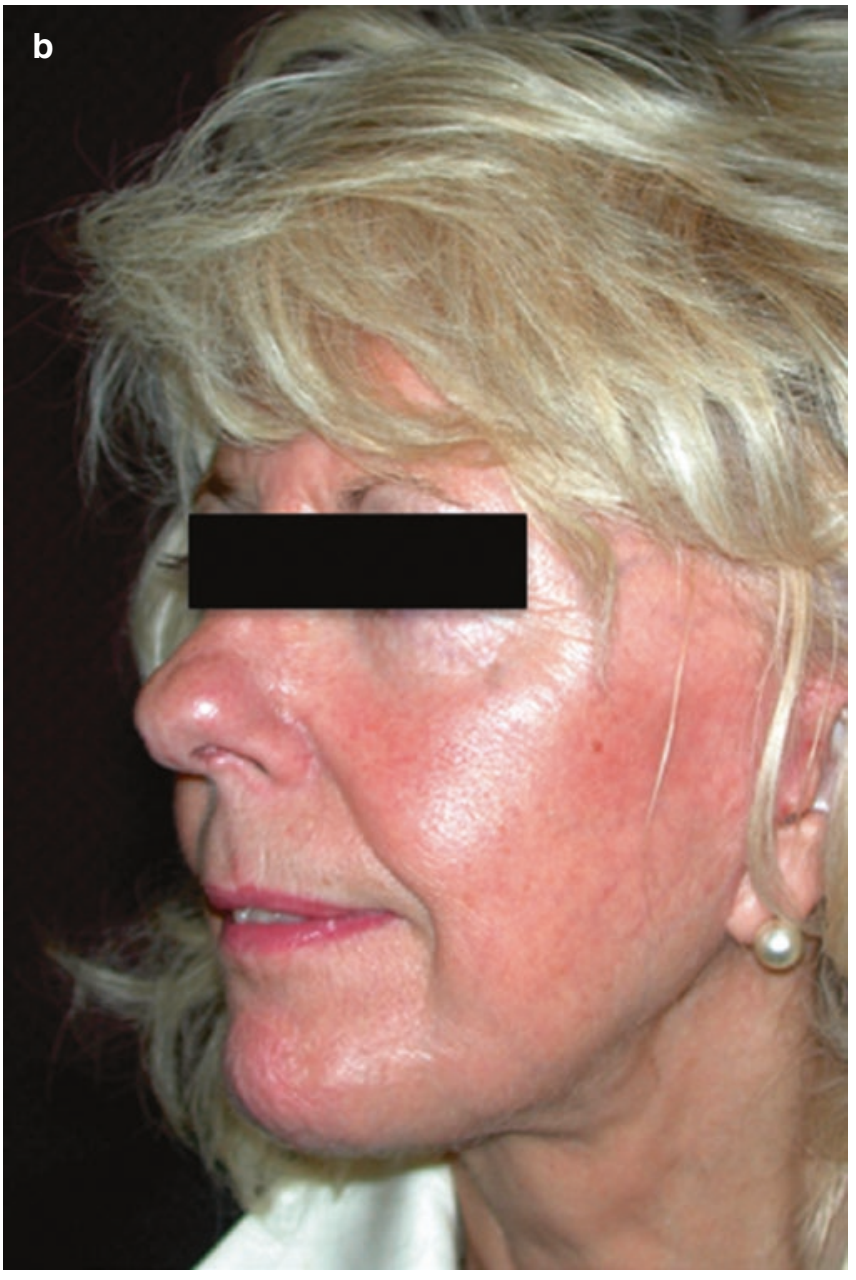

resected, jowls had been corrected and there was improved cervicomental definition

bed during which perfusion is only by the vessels supplying the flap. This causes the areas furthest away from the perfusing vessels to be at risk of ischaemia.

Necrosis of the skin is more common in the postauricular than the preauricular region.

Application of nitroglycerin paste may help to encourage vasodilation when done in the early stages [25]. Conservative treatment to allow healing via secondary intention followed by laser, excision of the scar with repair via rotation flaps can be carried out at a later stage. 

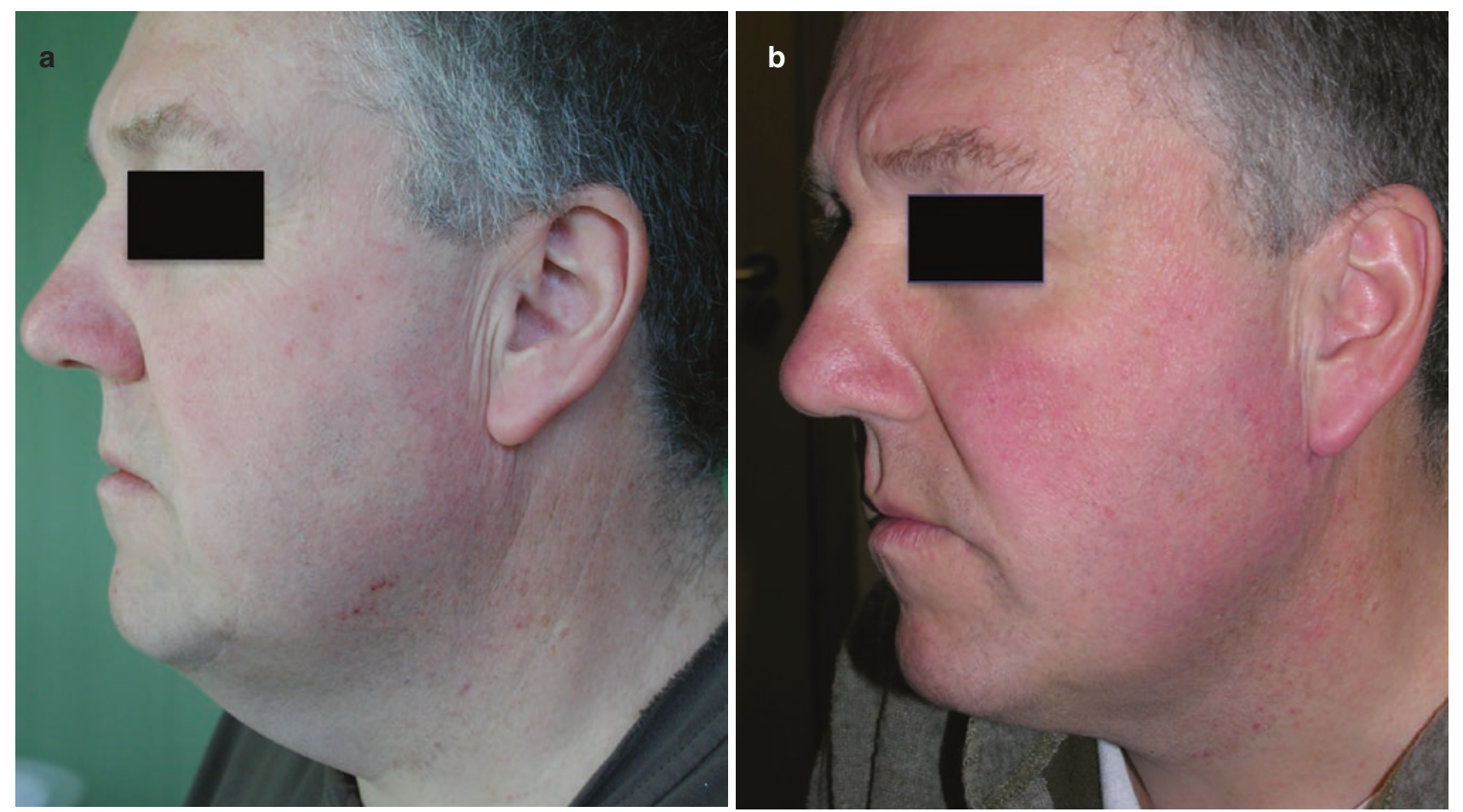

CAssociation of Oral and Maxillofacial Surgeons of India

Fig. 37.25 (a) Pre-operative profile view showing excess skin, slight jowling, submental fat and severe loss of neck definition. (b) Post-operative profile view after facelift and platysma plication

\subsubsection{Obvious Scarring}

Scarring can occur when the wounds are closed under excessive tension or if the incisions are placed at an inappropriate position due to poor design. Skin slough and necrosis of the flap can lead to severe scarring. Patients who are more prone to hypertrophic scarring or keloids should be warned. Wound tension can develop and cause scarring if there is excessive removal of skin or if undermining of the flap is inadequate.

Treatment of scarring includes conservative treatment with antibiotic ointment such as Tetracycline cream 3\% or Bactroban cream. This is followed by steroid injections such as triamcinolone acetate, application of silicone sheet or gel dressing. As the scar improves and stabilizes, it can be lasered, excised and revised.

\subsubsection{Sensory Disturbances}

Sensory disturbances occur invariably due to the dissection and separation of the facial planes during the facelift proce- dure, but this usually occurs temporarily and resolves in 1 year.

The most common sensory disturbance occurs to the great auricular nerve. The greater auricular nerve crosses the sternocleidomastoid muscle at approximately $6.5 \mathrm{~cm}$ inferior to the external auditory meatus and travels superiorly to supply the postauricular region.

Injury to this nerve can be temporary or permanent due to dissection over the postauricular, mastoid region and to a lesser extent in the cervical region. This results in numbness of the ear lobe and postauricular area.

Other forms of numbness can be found in preauricular region.

\subsubsection{Motor Nerve Disturbances}

Keeping away from the planes that the facial nerve travels in is the safest method to avoid motor nerve problems. The facial nerve branches course through the parotid gland and exits the parotid gland to travel deep to the SMAS layer before innervating the muscles of facial expression. Care 
should be taken when dissecting beyond the parotid or when dissecting deep into the SMAS layer.

Facial nerve injury ranges from 0.4 to $2.6 \%$ [21]. The most common motor nerve damage is the marginal mandibular nerve followed by the temporal nerve and then the buccal branch. Neuropraxia from exuberant retraction, cautery heat and compression can result in temporary or permanent loss of the motor function. The marginal mandibular branch courses in the superficial layer of the deep cervical fascia, deep into the platysmal layer, and may be damaged when dissecting in this region. The temporal branch of the facial nerve courses just deep to the superficial temporal fascia and travels $0.5 \mathrm{~cm}$ anterior to the tragus and then obliquely to $1.5 \mathrm{~cm}$ above the lateral edge of the eyebrows.

\subsubsection{Ear Lobe Irregularities}

"Pixie or elf-like" ear is a stigma of facelift. The ear lobe should be repositioned in a tensionless fashion, and the ear lobe should be placed $15^{\circ}$ posterior to the vertical axis of the pinna [6]. Over resection of the flap at the base of the auricle as well as failure to place subcutaneous stay sutures on the facial flap at the ear base can result in the ear being pulled inferiorly.

Pixie ears can be revised by releasing the base of the auricle and creating a new ear lobe.

\subsection{Conclusion}

The facelift remains the single most powerful method in facial rejuvenation for the moderate to advanced ageing face. It has developed from small elliptical incisions and closure to full facelift with simultaneous neck lift. A variety of techniques have been described contributing to its evolution including SMAS layer management, standard facelifts, composite facelifts, platysmal plication and smaller flaps such as the MACS lift and S-lifts. The standard face and simultaneous neck lift can treat most of the problems of the mid and lower face as well as the neck. An intimate understanding of the anatomy of the face, appropriate choice of the facelift design and meticulous execution are paramount in giving the patient an ideal outcome while avoiding the possible complications.

Disclosure Authors have no financial conflicts to disclose.

\subsection{Case Scenarios}

Case Scenario 1 (Fig. 37.26)

A 67-year-old Caucasian lady presented with unhappy aesthetics of her face. She had had a considerable amount of sun exposure over the years. Her medical history was unremarkable.

Clinical features:

On examination she had multiple minor and major wrinkles on her face. On examination of the mid face, she showed descending SOOF and malar fat pads. She had prominent folding of the nasolabial fold and loss of mandibular border definition. Her neck was a good shape for her age (Fig. 37.26a, b).

Treatment:

She underwent a standard facelift without a neck lift and simultaneous autologous fat augmentation malar, chin and perioral areas.

Post-operative findings:

The post-operative profile shows aesthetically pleasing contour with an unoperated look (Fig. 37.26c, d). A majority of ageing changes have been removed. She ideally should undergo perioral laser resurfacing to improve the skin quality.

\section{Case Scenario 2 (Fig. 37.27)}

A 73-old lady presented with ageing changes and was unhappy with the mid and lower face. She was concerned about the aesthetics of her neck (Fig. 37.27a, b).

She is an insulin-dependent diabetic and takes medication for hypertension.

Clinical features:

On examination, the mid face showed malar fat descent with prominent nasolabial fold. She had marked marionette lines, pre-jowl sulcus and prominent jowls. Examination of the lower face showed platysmal bands and loss of mandibular neck definition with submental fat projection.

Treatment:

She underwent a standard face and neck lift with platysmal and SMAS plication.

Post-operative findings:

Three month post-operative photograph shows correction for the ageing changes and a pleasing facial and neck profile (Fig. 37.27c, d). 

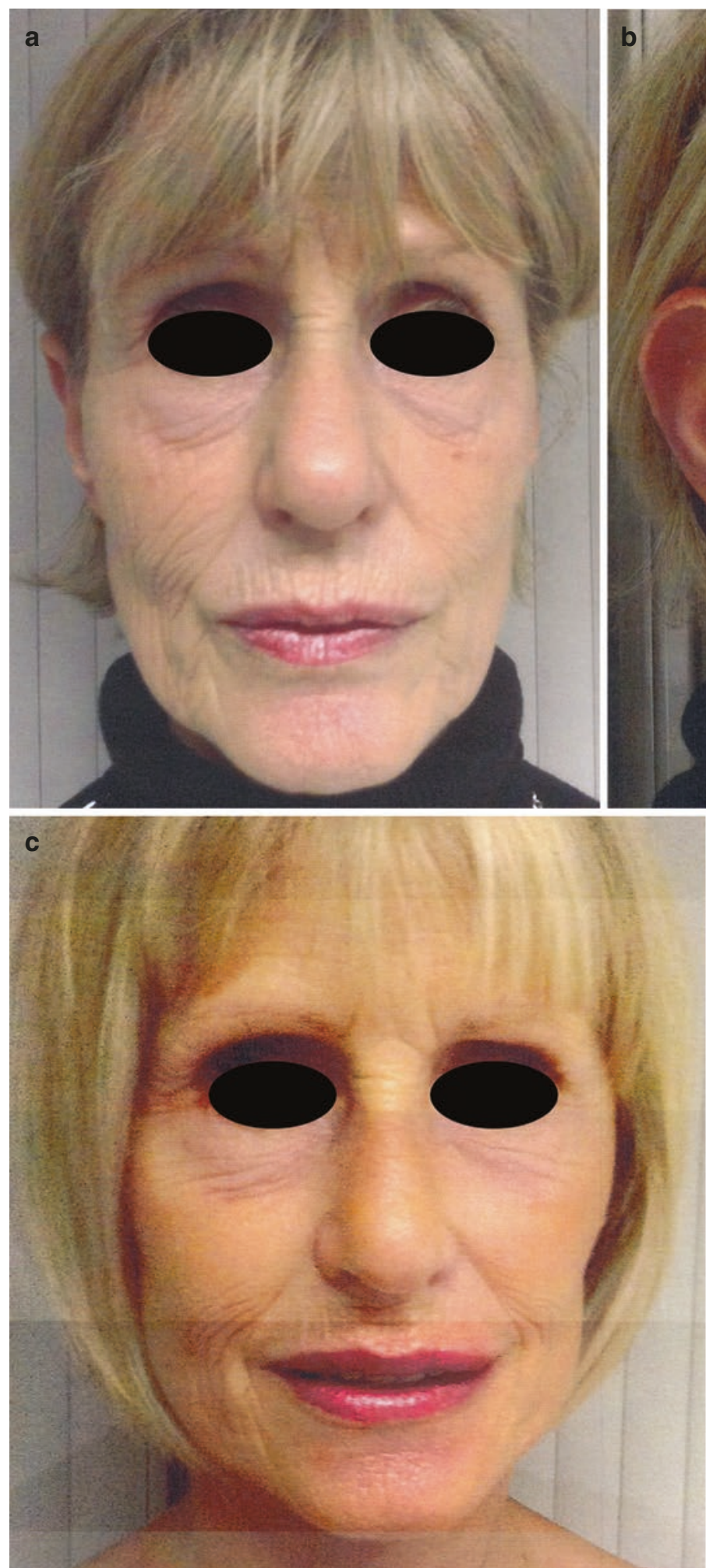

(C)Association of Oral and Maxillofacial Surgeons of India
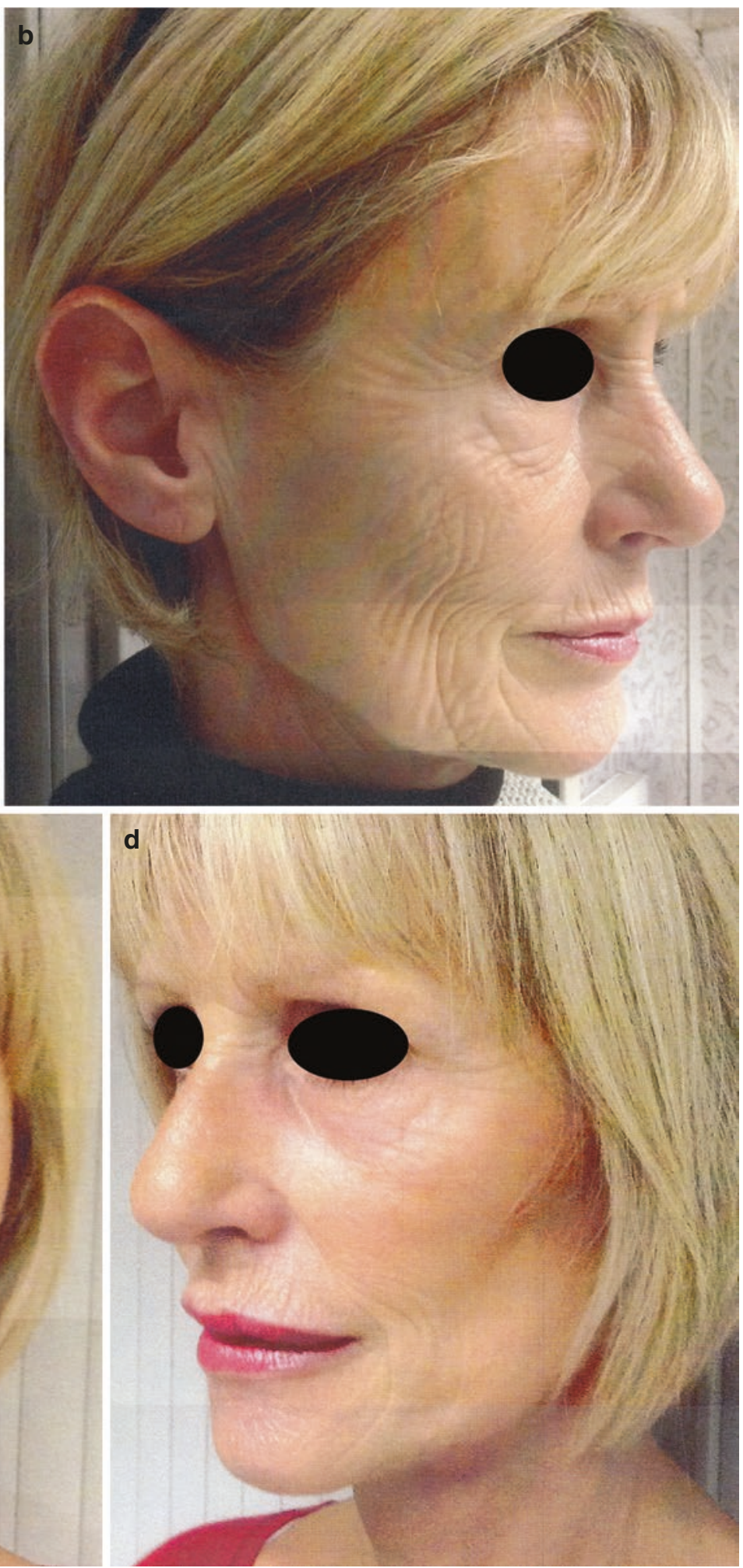

Fig. 37.26 Standard face and neck lift with platysmal and SMAS plication. (a, b) Pre-operative appearance demonstrating wrinkles in frontal and profile view. (c, d) Post-operative appearance demonstrating wrinkles in frontal and profile view 


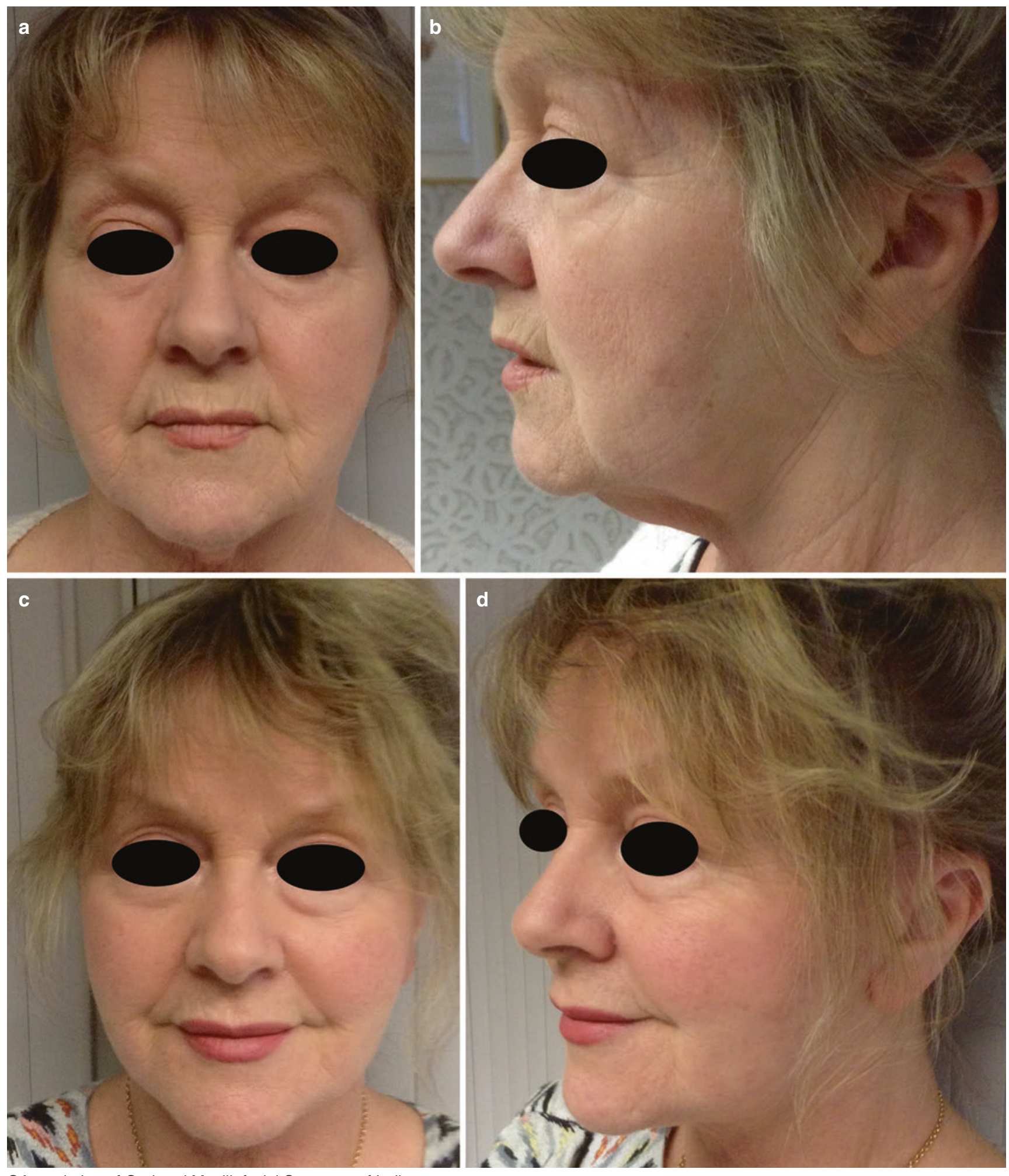

(C)Association of Oral and Maxillofacial Surgeons of India

Fig. 37.27 Standard face and neck lift with platysmal and SMAS plication. (a, b) Pre-operative appearance demonstrating wrinkles in frontal and profile view. (c, d) Post-operative appearance demonstrating wrinkles in frontal and profile view 


\section{References}

1. Ilankovan V. Anatomy of the aging face. Br J Oral Maxillofac Surg. 2014;52:195-202.

2. Hollander E. Die kosmetische chirurgie. In: Joseph M, editor. Handbuch der Kosmetik. Leipzig: Von Veit; 1912. p. 688.

3. Mangat DS, Frankel JK. The history of rhytidectomy. Facial Plast Surg. 2017;33(3):247-9. Epub 2017 Jun 1.

4. Tonnard PL, Verpaele AM. The MACS-lift short-scar rhytidectomy. Quality Medical Publishing; 2004. p. 4.

5. Mitz V, Peyronie M. The superficial musculoaponeurotic system (SMAS) in the parotid and cheek area. Plast Reconstr Surg. 1976;58(1):80-6.

6. Ilankovan V. Recent advances in face lift to achieve facial balance. J Maxillofac Oral Surg. 2017;16(1):3-12.

7. Webster RC, Smith RC, Papsidero MJ, Karolow WW, Smith KF. Comparison of SMAS plication with SMAS imbrication in face lifting. Laryngoscope. 1982;92(8):901-12.

8. Lemmon ML, Hamra ST. Skoog rhytidectomy: a five year experience with 577 patients. Plast Reconst Surg. 1980;65:283-97.

9. Baker DC. Lateral SMASectomy. Plast Reconstr Surg. 1997;100:509-13.

10. Hamra ST. Approaches to rejuvenation of the upper face. Aesthet Surg J. 2000;20(1):47-52.

11. Hamra ST. The role of the septal rest in creating a youthful eyelid-cheek complex in facial rejuvenation. Plast Reconstr Surg. 2004;113(7):2124-41.

12. Antell DE, Orseck MJ. A comparison of face lift techniques in eight consecutive sets of identical twins. Plast Reconstr Surg. 2007;120:1667-73.
13. Fulton JE, et al. The S-Lift facelift featuring the U-Suture and O-Suture combined with skin resurfacing. Dermatol Surg. 2001;27(1):18-22.

14. Tonnard P, et al. Minimal access cranial suspension lift: a modified S-Lift. Plas Reconstr Surg. 2002;109(6):2075-86.

15. Mendelson BC. Aesthetic plastic surgery. Saunders Elsevier; 2009. Chapter 6, pp. 53-71.

16. Warren RJ, et al. Face lift. Plast Reconstr Surg. 2011;128:747e-64e.

17. Stuzin JM, Baker TJ, Gordon HL, Baker TM. Extended SMAS dissection as an approach to midface rejuvenation. Clin Plast Surg. 1995;22(2):295-311.

18. Furnas DW. The retaining ligaments of the cheek. Plast Reconstr Surg. 1989;83:11-6.

19. Alghoul M, Codner MA. Retaining ligaments of the face: review of anatomy and clinical applications. Aesth Surg J. 2013;33(6):769-82.

20. Sebasan T, Ilankovan V. Plication of the platysma. Br J Oral Maxillofac Surg. 2006;44(4):325.

21. Bloom JD, et al. Face-lift complications. Facial Plast Surg. 2012;28:260-72.

22. Griffin JE, Jo C. Complications after superficial plane cervicofacial rhytidectomy: a retrospective analysis of 178 consecutive facelifts and review of the literature. J Oral Maxillofac Surg. 2007;65(11):2227-34.

23. Zoumalan R, Rizk SS. Hematoma rates I drainless deep-plane facelift surgery with and without the use of fibrin-glue. Arch Facial Plast Surg. 2008;10:103-7.

24. Baker DC. Complications of cervicofacial rhytidectomy. Clin Plast Surg. 1983;10:543-62.

25. Niamtu J. Cosmetic facial surgery: Elsevier Mosby; 2011. p. 354.

Open Access This chapter is licensed under the terms of the Creative Commons Attribution 4.0 International License (http://creativecommons. org/licenses/by/4.0/), which permits use, sharing, adaptation, distribution and reproduction in any medium or format, as long as you give appropriate credit to the original author(s) and the source, provide a link to the Creative Commons license and indicate if changes were made.

The images or other third party material in this chapter are included in the chapter's Creative Commons license, unless indicated otherwise in a credit line to the material. If material is not included in the chapter's Creative Commons license and your intended use is not permitted by statutory regulation or exceeds the permitted use, you will need to obtain permission directly from the copyright holder. 